Honam Mathematical J. 34 (2012), No. 4, pp. 533-548

http://dx.doi.org/10.5831/HMJ.2012.34.4.533

\title{
ON THE RECURRENCE SEQUENCES
}

\author{
Eunmi ChOI
}

\begin{abstract}
We investigate relations of polynomials $x^{n}=\sum_{i=0}^{n-1} x^{i}$ and recurrence sequences. We consider certain variations of the Fibonacci sequence and investigate explicit ways to compute the general $n$th term.
\end{abstract}

\section{Introduction}

The Fibonacci sequence $F_{n}=F_{n-1}+F_{n-2}(n \geq 2)$ with $F_{0}=0$ and $F_{1}=1$ is well connected to $f(x)=x^{2}-x-1$. A variation of the Fibonacci sequence that begins with $L_{0}=2, L_{1}=1$ and $L_{n}=$ $L_{n-1}+L_{n-2}(n \geq 2)$ is called the Lucas sequence. The ratio $L_{n} / L_{n-1}$ satisfies $x^{2}-x-1=0$ for large enough $n$, and it meets $W_{n}=A W_{n-1}$ with $W_{n}=\left[\begin{array}{c}L_{n} \\ L_{n-1}\end{array}\right]$ and $A=\left[\begin{array}{ll}1 & 1 \\ 1 & 0\end{array}\right]$. Since the characteristic polynomial $x^{2}-x-1$ of $A$ has two roots $\phi_{i}$ with eigenvectors $\left[\begin{array}{c}\phi_{i} \\ 1\end{array}\right], A$ is diagonalized to $D=\left[\begin{array}{cc}\phi_{1} & 0 \\ 0 & \phi_{2}\end{array}\right]$ with $P=\left[\begin{array}{cc}\phi_{1} & \phi_{2} \\ 1 & 1\end{array}\right]$, so $L_{n}=\phi_{1}^{n}+\phi_{2}^{n}=\phi_{1}^{n}+\left(-\phi_{1}\right)^{-n}$. This shows that both Fibonacci and Lucas sequences are represented by $f(x)=x^{2}-x-1$, and their $n$th terms are determined by diagonalization of matrix whose eigenvalues are the zeros of $f(x)$. The purpose of this work is to investigate interrelationships of polynomials and recurrence sequences. We will study certain variations of Fibonacci sequence and find concrete methods to calculate general $n$th terms.

Received August 25, 2012. Accepted September 22, 2012.

2010 Mathematics Subject Classification. 11D09, 12F10, $20 \mathrm{~B} 05$.

Key words and phrases. recurrence sequence, fibonacci sequence, resultant.

This work is supported by HanNam University Research fund 2012. 


\section{Variations of Fibonacci sequence}

The relations of $L_{n}$ and $\phi_{i}$ can also be easily obtained by iteration of recurrence.

Lemma 1. $\phi_{1}^{n}-\phi_{2}^{n}=\sqrt{5} F_{n}$ and $\phi_{1}^{n}+\phi_{2}^{n}=L_{n}$ for all $n \in \mathbb{Z}$.

A generalization of Fibonacci sequence is as follows.

Theorem 2. Let $M_{n}=c_{1} M_{n-1}+c_{2} M_{n-2}\left(n \geq 2, c_{i} \in \mathbb{R}\right)$ with $M_{0}=a, M_{1}=b$. If $\lambda$ is a root of $x^{2}-c_{1} x-c_{2}$ and $c_{1}^{2}+4 c_{2} \neq 0$, then the $n$th term $M_{n}$ is

$$
M_{n}=\frac{b\left(\lambda^{n}-\left(-c_{2} \lambda^{-1}\right)^{n}\right)+a c_{2}\left(\lambda^{n-1}-\left(-c_{2} \lambda^{-1}\right)^{n-1}\right)}{\sqrt{c_{1}^{2}+4 c_{2}}} .
$$

Proof. Let $W_{n}=\left[\begin{array}{c}M_{n} \\ M_{n-1}\end{array}\right]$. Then $W_{n}=A W_{n-1}=A^{n-1}\left[\begin{array}{l}b \\ a\end{array}\right]$ with $A=\left[\begin{array}{cc}c_{1} & c_{2} \\ 1 & 0\end{array}\right]$. The characteristic polynomial of $A$ is $x^{2}-c_{1} x-c_{2}$ having two roots $\lambda_{i}$, whose eigenvectors are $\left[\begin{array}{c}\lambda_{i} \\ 1\end{array}\right]$. So $A$ is diagonalized to $D=\left[\begin{array}{cc}\lambda_{1} & 0 \\ 0 & \lambda_{2}\end{array}\right]$ with $P=\left[\begin{array}{cc}\lambda_{1} & \lambda_{2} \\ 1 & 1\end{array}\right]$, and

$$
\begin{aligned}
{\left[\begin{array}{c}
M_{n} \\
M_{n-1}
\end{array}\right]=W_{n} } & =\left[\begin{array}{cc}
\lambda_{1} & \lambda_{2} \\
1 & 1
\end{array}\right]\left[\begin{array}{cc}
\lambda_{1}^{n-1} & 0 \\
0 & \lambda_{2}^{n-1}
\end{array}\right]\left[\begin{array}{cc}
\lambda_{1} & \lambda_{2} \\
1 & 1
\end{array}\right]^{-1}\left[\begin{array}{l}
b \\
a
\end{array}\right] \\
& =\frac{1}{\lambda_{1}-\lambda_{2}}\left[\begin{array}{c}
b\left(\lambda_{1}^{n}-\lambda_{2}^{n}\right)-a\left(\lambda_{1}^{n} \lambda_{2}-\lambda_{1} \lambda_{2}^{n}\right) \\
b\left(\lambda_{1}^{n-1}-\lambda_{2}^{n-1}\right)-a\left(\lambda_{1}^{n-1} \lambda_{2}-\lambda_{1} \lambda_{2}^{n-1}\right)
\end{array}\right] .
\end{aligned}
$$

Since $\lambda_{1}+\lambda_{2}=c_{1}, \lambda_{1} \lambda_{2}=-c_{2}$ and $\lambda_{1}-\lambda_{2}=\sqrt{c_{1}^{2}+4 c_{2}}$, we have

$$
\begin{aligned}
M_{n} & =b \frac{\lambda_{1}^{n}-\lambda_{2}^{n}}{\lambda_{1}-\lambda_{2}}-a \lambda_{1} \lambda_{2} \frac{\lambda_{1}^{n-1}-\lambda_{2}^{n-1}}{\lambda_{1}-\lambda_{2}}=b \frac{\lambda_{1}^{n}-\lambda_{2}^{n}}{\lambda_{1}-\lambda_{2}}+a c_{2} \frac{\lambda_{1}^{n-1}-\lambda_{2}^{n-1}}{\lambda_{1}-\lambda_{2}} \\
& =\frac{b\left(\lambda^{n}-\left(-c_{2} \lambda^{-1}\right)^{n}\right)+a c_{2}\left(\lambda^{n-1}-\left(-c_{2} \lambda^{-1}\right)^{n-1}\right)}{\sqrt{c_{1}^{2}+4 c_{2}}} \cdot \square
\end{aligned}
$$

We briefly review the resultant and discriminant ([3]). Let $r_{i}(1 \leq i \leq$ $n)$ and $s_{j}(1 \leq j \leq m)$ be roots of $f(x)=\sum_{i=0}^{n} a_{i} x^{i}, g(x)=\sum_{j=0}^{m} b_{j} x^{j}$ respectively in a splitting field. The resultant $R(f, g)$ and the discriminant 
$\Delta(f)$ are defined by

$$
R(f, g)=a_{n}^{m} b_{m}^{n} \prod_{i=1}^{n} \prod_{j=1}^{m}\left(r_{i}-s_{j}\right) \text { and } \Delta(f)=a_{n}^{2 n-2} \prod_{1 \leq i<j \leq n}\left(r_{i}-r_{j}\right)^{2} .
$$

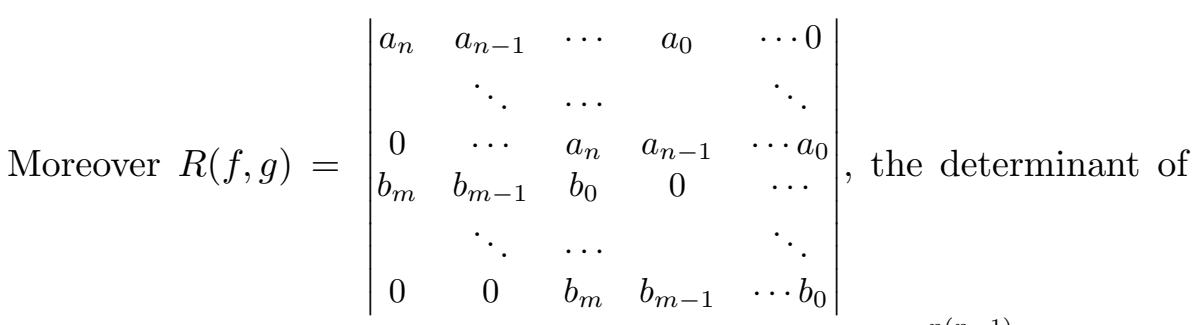

$(n+m) \times(n+m)$ Sylvester matrix. And $\Delta(f)=(-1)^{\frac{n(n-1)}{2}} \frac{1}{a_{n}} R\left(f, f^{\prime}\right)$ where $f^{\prime}$ is the derivative of $f$, so $\Delta(f) \neq 0$ if and only if $f(x)$ has all distinct roots. Thus $\Delta(f)$ provide a criteria if $f$ has a multiple root, while $R(f, g)$ is used to verify if $f, g$ have a common root. Let $A_{n}$ be a sequence with $t$ predetermined terms $A_{0}=\ldots=A_{t-2}=0, A_{t-1}=1$ and each term is the sum of preceding $t$ terms

$$
A_{n}=A_{n-1}+A_{n-2}+\cdots+A_{n-t}(n \geq t) .
$$

Theorem 3. The $A_{n+1} / A_{n}$ satisfies the characteristic equation $f(x)$ of $t \times t$ matrix $\left[\begin{array}{ccccc}1 & 1 & \cdots & 1 & 1 \\ 1 & 0 & \ddots & \cdots & 0 \\ 0 & 0 & \cdots & 1 & 0\end{array}\right]$. If $\lambda_{i}$ are roots of $f(x)$ and $\mu=$ $\prod_{1 \leq i<j \leq t}\left(\lambda_{i}-\lambda_{j}\right)$ then

$$
A_{n}=\frac{1}{\mu} \sum_{i=1}^{t} \lambda_{i}^{n} \prod_{j, k \neq i}^{1 \leq j<k \leq t}\left(\lambda_{j}-\lambda_{k}\right)=\sum_{i=1}^{t} \frac{\lambda_{i}^{n}}{\prod_{i \neq j=1}^{t}\left(\lambda_{i}-\lambda_{j}\right)} .
$$

Proof. We first assume $t=3$. As $n$ gets larger the ratio

$$
\frac{A_{n}}{A_{n-1}}=\frac{A_{n-1}+A_{n-2}+A_{n-3}}{A_{n-1}}=1+\frac{A_{n-2}}{A_{n-1}}+\frac{A_{n-3} / A_{n-2}}{A_{n-1} / A_{n-2}}
$$

satisfies $x=1+\frac{1}{x}+\frac{1}{x^{2}}$, i.e., $f(x)=x^{3}-x^{2}-x-1$. Let $M=\left[\begin{array}{lll}1 & 1 & 1 \\ 1 & 0 & 0 \\ 0 & 1 & 0\end{array}\right]$ and $Y_{n}=\left[\begin{array}{c}A_{n} \\ A_{n-1} \\ A_{n-2}\end{array}\right]$. Then $Y_{n}=M Y_{n-1}=M^{n-2}\left[\begin{array}{l}1 \\ 0 \\ 0\end{array}\right]$, and $f(x)$ is the 
characteristic polynomial of $M$ having all distinct roots $\lambda_{i}$, because

$$
\Delta(f)=(-1)^{\frac{3 \cdot 2}{2}} \operatorname{Res}\left(f, f^{\prime}\right)=-\left|\begin{array}{ccccc}
1 & -1 & -1 & -1 & 0 \\
0 & 1 & -1 & -1 & -1 \\
3 & -2 & -1 & 0 & 0 \\
0 & 3 & -2 & -1 & 0 \\
0 & 0 & 3 & -2 & -1
\end{array}\right|=-44 \neq 0 .
$$

Each $\lambda_{i}$ is an eigenvalue of $M$ with corresponding eigenvector $\left[\begin{array}{c}\lambda_{i}^{2} \\ \lambda_{i} \\ 1\end{array}\right]$, so $M$ can be diagonalized to $D=\left[\begin{array}{ccc}\lambda_{1} & 0 & 0 \\ 0 & \lambda_{2} & 0 \\ 0 & 0 & \lambda_{3}\end{array}\right]$ with $P=\left[\begin{array}{ccc}\lambda_{1}^{2} & \lambda_{2}^{2} & \lambda_{3}^{2} \\ \lambda_{1} & \lambda_{2} & \lambda_{3} \\ 1 & 1 & 1\end{array}\right]$.

Hence

$$
\begin{aligned}
& {\left[\begin{array}{c}
A_{n} \\
A_{n-1} \\
A_{n-2}
\end{array}\right]=\left[\begin{array}{ccc}
\lambda_{1}^{2} & \lambda_{2}^{2} & \lambda_{3}^{2} \\
\lambda_{1} & \lambda_{2} & \lambda_{3} \\
1 & 1 & 1
\end{array}\right]\left[\begin{array}{ccc}
\lambda_{1}^{n-2} & 0 & 0 \\
0 & \lambda_{2}^{n-2} & 0 \\
0 & 0 & \lambda_{3}^{n-2}
\end{array}\right]\left[\begin{array}{ccc}
\lambda_{1}^{2} & \lambda_{2}^{2} & \lambda_{3}^{2} \\
\lambda_{1} & \lambda_{2} & \lambda_{3} \\
1 & 1 & 1
\end{array}\right]^{-1}\left[\begin{array}{l}
1 \\
0 \\
0
\end{array}\right]} \\
& =\frac{1}{\mu}\left[\begin{array}{c}
\lambda_{1}^{n}\left(\lambda_{2}-\lambda_{3}\right)-\lambda_{2}^{n}\left(\lambda_{1}-\lambda_{3}\right)+\lambda_{3}^{n}\left(\lambda_{1}-\lambda_{2}\right) \\
\lambda_{1}^{n-1}\left(\lambda_{2}-\lambda_{3}\right)-\lambda_{2}^{n-1}\left(\lambda_{1}-\lambda_{3}\right)+\lambda_{3}^{n-1}\left(\lambda_{1}-\lambda_{2}\right) \\
\lambda_{1}^{n-2}\left(\lambda_{2}-\lambda_{3}\right)-\lambda_{2}^{n-2}\left(\lambda_{1}-\lambda_{3}\right)+\lambda_{3}^{n-2}\left(\lambda_{1}-\lambda_{2}\right)
\end{array}\right]
\end{aligned}
$$

where $\mu=\prod_{1 \leq i<j \leq 3}\left(\lambda_{i}-\lambda_{j}\right)=\operatorname{det}(P)$, the determinant of $P$ due to Vandermonde. We note that $\Delta \neq 0$ so $\mu \neq 0$. Thus

$$
A_{n}=\frac{1}{\mu}\left(\lambda_{1}^{n}\left(\lambda_{2}-\lambda_{3}\right)-\lambda_{2}^{n}\left(\lambda_{1}-\lambda_{3}\right)+\lambda_{3}^{n}\left(\lambda_{1}-\lambda_{2}\right)\right)=\sum_{i=1}^{3} \frac{\lambda_{i}^{n}}{\prod_{i \neq j=1}^{3}\left(\lambda_{i}-\lambda_{j}\right)} .
$$

Similarly if $t=4$, then for the large enough $n$, $\frac{A_{n+1}}{A_{n}}=1+\frac{A_{n-1}}{A_{n}}+\frac{A_{n-2} / A_{n-1}}{A_{n} / A_{n-1}}+\frac{\left(A_{n-3} / A_{n-2}\right) /\left(A_{n-1} / A_{n-2}\right)}{A_{n} / A_{n-1}}$ satisfies $x=$ $1+\frac{1}{x}+\frac{1}{x^{2}}+\frac{1}{x^{3}}$, so the ratio is a root of $f(x)=x^{4}-x^{3}-x^{2}-x-1$. Moreover

$$
Y_{n}=M Y_{n-1}=M^{n-3} Y_{3} \quad \text { with } M=\left[\begin{array}{llll}
1 & 1 & 1 & 1 \\
1 & 0 & 0 & 0 \\
0 & 1 & 0 & 0 \\
0 & 0 & 1 & 0
\end{array}\right], Y_{n}=\left[\begin{array}{c}
B_{n} \\
B_{n-1} \\
B_{n-2} \\
B_{n-3}
\end{array}\right]
$$

and $f(x)$ is the characteristic polynomial of $M$ with all distinct roots $\lambda_{i}$, for 
$\Delta(f)=(-1)^{\frac{4 \cdot 3}{2}} \operatorname{Res}\left(f, f^{\prime}\right)=\left|\begin{array}{ccccccc}1 & -1 & -1 & -1 & -1 & 0 & 0 \\ 0 & 1 & -1 & -1 & -1 & -1 & 0 \\ 0 & 0 & 1 & -1 & -1 & -1 & -1 \\ 4 & -3 & -2 & -1 & 0 & 0 & 0 \\ 0 & 4 & -3 & -2 & -1 & 0 & 0 \\ 0 & 0 & 4 & -3 & -2 & -1 & 0 \\ 0 & 0 & 0 & 4 & -3 & -2 & -1\end{array}\right|=-563 \neq$ 0.

Each $\lambda_{i}(1 \leq i \leq 4)$ corresponds to eigenvector $\left[\begin{array}{c}\lambda_{i}^{3} \\ \lambda_{i}^{2} \\ \lambda_{i} \\ 1\end{array}\right]$, so $M$ can be diagonalized to $D=\left[\begin{array}{cccc}\lambda_{1} & 0 & 0 & 0 \\ 0 & \lambda_{2} & 0 & 0 \\ 0 & 0 & \lambda_{3} & 0 \\ 0 & 0 & 0 & \lambda_{4}\end{array}\right]$ with $P=\left[\begin{array}{cccc}\lambda_{1}^{3} & \lambda_{2}^{3} & \lambda_{3}^{3} & \lambda_{4}^{3} \\ \lambda_{1}^{2} & \lambda_{2}^{2} & \lambda_{3}^{2} & \lambda_{4}^{2} \\ \lambda_{1} & \lambda_{2} & \lambda_{3} & \lambda_{4} \\ 1 & 1 & 1 & 1\end{array}\right]$. Hence

$$
Y_{n}=M^{n-3} Y_{3}=\frac{1}{\mu}\left[\begin{array}{c}
\sum_{k=1}^{4} \lambda_{k}^{n} \prod_{i, j \neq k}^{i<j}\left(\lambda_{i}-\lambda_{j}\right) \\
\sum_{k=1}^{4} \lambda_{k}^{n-1} \prod_{i, j=k}^{i<j}\left(\lambda_{i}-\lambda_{j}\right) \\
\sum_{k=1}^{4} \lambda_{k}^{n-2} \prod_{i, j \neq k}^{i<j}\left(\lambda_{i}-\lambda_{j}\right) \\
\sum_{k=1}^{4} \lambda_{k}^{n-3} \prod_{i, j \neq k}^{i<j}\left(\lambda_{i}-\lambda_{j}\right)
\end{array}\right]
$$

with $\mu=\prod_{1 \leq i<j \leq 4}\left(\lambda_{i}-\lambda_{j}\right)=\operatorname{det}(P)$. Therefore it follows that

$$
A_{n}=\frac{1}{\mu} \sum_{i=1}^{4} \lambda_{i}^{n} \prod_{i \neq j, k}^{1 \leq j<k \leq 4}\left(\lambda_{j}-\lambda_{k}\right)=\sum_{i=1}^{4} \frac{\lambda_{i}^{n}}{\prod_{i \neq j=1}^{4}\left(\lambda_{i}-\lambda_{j}\right)} .
$$

Assume $t>4$. It is not hard to see the ratio $\frac{A_{n+1}}{A_{n}}$ satisfies $x^{t}-x^{t-1}-$ $\cdots-1=0$, and the sequence $A_{n}$ yields the $t \times t$ matrix $M_{t}=\left[\begin{array}{cccc}1 & 1 & \cdots & 1 \\ 1 & 0 & \cdots & 0 \\ 0 & \ddots & 1 & 0\end{array}\right]$. For characteristic polynomial $\operatorname{char}\left(M_{t}\right)$ of $M_{t}$, claim that

$$
\operatorname{char}\left(M_{t}\right)=\left|\lambda I-M_{t}\right|=\lambda^{t}-\lambda^{t-1}-\cdots-\lambda-1 .
$$

Obviously, $\operatorname{char}\left(M_{2}\right)=\left|\lambda I-M_{2}\right|=\left|\begin{array}{cc}\lambda-1 & -1 \\ -1 & \lambda\end{array}\right|=\lambda^{2}-\lambda-1$ and $\begin{aligned} \operatorname{char}\left(M_{3}\right) & =\left|\lambda I-M_{3}\right|=\left|\begin{array}{ccc}\lambda-1 & -1 & -1 \\ -1 & \lambda & 0 \\ 0 & -1 & \lambda\end{array}\right|=\left|\begin{array}{cc}\lambda-1 & -1 \\ -1 & 0\end{array}\right|+\lambda\left|\begin{array}{cc}\lambda-1 & -1 \\ -1 & \lambda\end{array}\right| \\ & =-1+\lambda\left|\lambda I-M_{2}\right|=\lambda^{3}-\lambda^{2}-\lambda-1 .\end{aligned}$ Assume $\operatorname{char}\left(M_{i}\right)=\lambda^{i}-\cdots-\lambda-1$ for all $i \leq t-1$. Then $\operatorname{char}\left(M_{t}\right)$ equals 
$\left|\begin{array}{ccccc}\lambda-1 & -1 & \cdots & -1 & -1 \\ -1 & \lambda & 0 & 0 & 0 \\ 0 & -1 & \lambda & \cdots & 0 \\ 0 & 0 & \ddots & -1 & \lambda\end{array}\right|=\left|\begin{array}{cccc}\lambda-1 & -1 & \cdots & -1 \\ -1 & \lambda & \cdots & 0 \\ 0 & -1 & \ddots & 0 \\ 0 & \cdots & -1 & 0\end{array}\right|+\lambda\left|\begin{array}{ccccc}\lambda-1 & -1 & \cdots & -1 & -1 \\ -1 & \lambda & \cdots & 0 & 0 \\ 0 & -1 & \lambda & \ddots & 0 \\ 0 & \cdots & 0 & -1 & \lambda\end{array}\right|$

where the former one of the last equation equals $\left|\begin{array}{cc}\lambda-1 & -1 \\ -1 & 0\end{array}\right|=-1$ while the latter one is $\left|\lambda I-M_{t-1}\right|=\lambda^{t-1}-\cdots-\lambda-1$. Hence

$$
\operatorname{char}\left(M_{t}\right)=-1+\lambda\left(\lambda^{t-1}-\cdots-\lambda-1\right)=\lambda^{t}-\lambda^{t-1}-\cdots-\lambda-1,
$$

has distinct roots $\lambda_{i}(1 \leq i \leq t)$. Thus for $\mu=\prod_{1 \leq i<j \leq t}\left(\lambda_{i}-\lambda_{j}\right), A_{n}$ equals

$$
A_{n}=\frac{1}{\mu} \sum_{i=1}^{t} \lambda_{i}^{n} \prod_{i \neq j, k}^{j<k}\left(\lambda_{j}-\lambda_{k}\right)=\sum_{i=1}^{t} \frac{\lambda_{i}^{n}}{\prod_{i \neq j=1}^{t}\left(\lambda_{i}-\lambda_{j}\right)}
$$

\section{General linear homogeneous recurrence sequence}

We have seen that the sequence $A_{n}=A_{n-1}+A_{n-2}+\cdots+A_{n-t}$ $(n \geq t)$ with $A_{0}=\cdots=A_{t-2}=0, A_{t-1}=1$ is related to $f(x)=$ $x^{t}-x^{t-1}-\cdots-1$, and the $n$th $A_{n}$ is represented by diagonalization of $t \times t$ matrix. Practically, however it is not useful for large $t$. For $c_{i} \in \mathbb{R}$, set $f(x)=x^{t}-c_{1} x^{t-1}-\cdots-c_{t}$ with roots $r_{i}(1 \leq i \leq t)$ and consider a general linear homogeneous recurrence relation

$$
\left\{A_{n}\right\}: A_{n}=c_{1} A_{n-1}+c_{2} A_{n-2}+\cdots+c_{t} A_{n-t}(n \geq t) .
$$

Lemma 4. [4] With the above $\left\{A_{n}\right\}$ and $f(x)$, if $f(x)$ has all distinct roots $r_{i}$ then $A_{n}$ is a linear combination $\sum_{i=1}^{t} \alpha_{i} r_{i}^{n}\left(\alpha_{i} \in \mathbb{R}\right)$ of $r_{i}$.

The $f(x)$ is called the characteristic polynomial of $\left\{A_{n}\right\}$. Lemma 4 yields Lemma 1 immediately that the characteristic polynomial of $F_{n}=F_{n-1}+F_{n-2}$ is $f(x)=x^{2}-x-1$. If $\lambda_{1}, \lambda_{2}$ are two real roots of $f(x)$, then $F_{n}$ can be written as $F_{n}=\alpha_{1} \lambda_{1}^{n}+\alpha_{2} \lambda_{2}^{n}$. Since $0=F_{0}=\alpha_{1}+\alpha_{2}$ and $1=F_{1}=\alpha_{1} \lambda_{1}+\alpha_{2} \lambda_{2}$, we have $\alpha_{1}=1 /\left(\lambda_{1}-\lambda_{2}\right)$ and $\alpha_{2}=-1 /\left(\lambda_{1}-\lambda_{2}\right)$, so $F_{n}=\frac{1}{\lambda_{1}-\lambda_{2}}\left(\lambda_{1}^{n}-\lambda_{2}^{n}\right)$.

Instead of finding roots of high degree polynomial, it might be at least useful to determine whether polynomial has distinct roots.

Lemma 5. The polynomials $f(x)=x^{3}-x^{2}-x-1, g(x)=x^{4}-$ $x^{3}-x^{2}-x-1$ and $h(x)=x^{5}-x^{4}-x^{3}-x^{2}-x-1$ have no multiple roots. 
Proof. $\Delta(f)=-44 \neq 0$, so the roots of $f(x)$ are all distinct by Lemma 3. Similarly

$$
\begin{aligned}
\Delta(g) & =\left|\begin{array}{ccccccc}
1 & -1 & -1 & -1 & -1 & 0 & 0 \\
0 & 1 & -1 & -1 & -1 & -1 & 0 \\
0 & 0 & 1 & -1 & -1 & -1 & -1 \\
4 & -3 & -2 & -1 & 0 & 0 & 0 \\
0 & 4 & -3 & -2 & -1 & 0 & 0 \\
0 & 0 & 4 & -3 & -2 & -1 & 0 \\
0 & 0 & 0 & 4 & -3 & -2 & -1
\end{array}\right| \\
& =\left|\begin{array}{ccccccc}
1 & -1 & -1 & -1 & -1 & 0 & 0 \\
0 & 1 & -1 & -1 & -1 & -1 & 0 \\
0 & 0 & 1 & -1 & -1 & -1 & -1 \\
0 & 0 & 0 & 7 & 8 & 4 & 3 \\
0 & 0 & 0 & 3 & 4 & 5 & 1 \\
0 & 0 & 0 & 1 & 2 & 3 & 4 \\
0 & 0 & 0 & 4 & -3 & -2 & -1
\end{array}\right| \\
& =\left|\begin{array}{cccc|l}
7 & 8 & 4 & 3 \\
3 & 4 & 5 & 1 \\
1 & 2 & 3 & 4 \\
4 & -3 & -2 & -1
\end{array}\right|=-563 \neq 0
\end{aligned}
$$

and

$$
\begin{aligned}
\Delta(h) & =\left|\begin{array}{ccccccccc}
1-1 & -1-1 & -1 & -1 & 0 & 0 & 0 \\
0 & 1 & -1-1 & -1 & -1 & -1 & 0 & 0 \\
0 & 0 & 1-1 & -1 & -1 & -1 & -1 & 0 \\
0 & 0 & 0 & 1 & -1 & -1 & -1 & -1 & -1 \\
0 & 0 & 0 & 0 & 15 & 16 & 11 & 10 & 7 \\
0 & 0 & 0 & 0 & 7 & 8 & 9 & 4 & 3 \\
0 & 0 & 0 & 0 & 3 & 4 & 5 & 6 & 1 \\
0 & 0 & 0 & 0 & 1 & 2 & 3 & 4 & 5 \\
0 & 0 & 0 & 0 & 5 & -4 & -3 & -2 & -1
\end{array}\right| \\
& =\left|\begin{array}{ccccc}
15 & 16 & 11 & 10 & 7 \\
7 & 8 & 9 & 4 & 3 \\
3 & 4 & 5 & 6 & 1 \\
1 & 2 & 3 & 4 & 5 \\
5 & -4 & -3 & -2 & -1
\end{array}\right|=9584 \neq 0
\end{aligned}
$$

, so $g(x)$ and $h(x)$ have all distinct roots.

Theorem 6. Let $\left\{A_{n}\right\}: A_{n}=A_{n-1}+A_{n-2}+A_{n-3}$ with $A_{0}=A_{1}=0$ and $A_{2}=1$. Let $\lambda_{i}(i=1,2,3)$ be the roots of characteristic polynomial 
$f(x)$ of $\left\{A_{n}\right\}$. Then

$$
A_{n}=\sum_{i=1}^{3} \frac{\lambda_{i}^{n}}{\prod_{i \neq j=1}^{3}\left(\lambda_{i}-\lambda_{j}\right)}=\frac{1}{3^{n}} \frac{\left(\tau+\frac{4}{\tau}+1\right)^{n+1}}{\left(\tau+\frac{4}{\tau}+2\right)\left(\tau+\frac{4}{\tau}-2\right)}
$$

for $\tau=\sqrt[3]{19+3 \sqrt{33}}$. Moreover $A_{n} \sim \lambda_{1}^{n} /\left(\lambda_{1}-\lambda_{2}\right)\left(\lambda_{1}-\lambda_{3}\right)$ asymptotically.

Proof. Due to Lemma 4, the characteristic polynomial of $\left\{A_{n}\right\}$ is $f(x)=x^{3}-x^{2}-x-1$, and $A_{n}=\alpha_{1} \lambda_{1}^{n}+\alpha_{2} \lambda_{2}^{n}+\alpha_{3} \lambda_{3}^{n}$ with $\alpha_{i} \in \mathbb{R}$. The $\alpha_{i}$ is determined by initial values $0=A_{0}=A_{1}$ and $1=A_{2}$ so we have

$$
\left[\begin{array}{ccc|c}
1 & 1 & 1 & 0 \\
\lambda_{1} & \lambda_{2} & \lambda_{3} & 0 \\
\lambda_{1}^{2} & \lambda_{2}^{2} & \lambda_{3}^{2} & 1
\end{array}\right]=\left[\begin{array}{ccc|c}
1 & 1 & 1 & 0 \\
0 & \lambda_{2}-\lambda_{1} & \lambda_{3}-\lambda_{1} & 0 \\
0 & 0 & \left(\lambda_{3}-\lambda_{1}\right)\left(\lambda_{3}-\lambda_{2}\right) & 1
\end{array}\right],
$$

hence $\alpha_{i}=\frac{1}{\left(\lambda_{i}-\lambda_{j}\right)\left(\lambda_{i}-\lambda_{k}\right)}$, thus

$$
\begin{aligned}
A_{n} & =\frac{\lambda_{1}^{n}\left(\lambda_{2}-\lambda_{3}\right)-\lambda_{2}^{n}\left(\lambda_{1}-\lambda_{3}\right)+\lambda_{3}^{n}\left(\lambda_{1}-\lambda_{2}\right)}{\left(\lambda_{1}-\lambda_{2}\right)\left(\lambda_{1}-\lambda_{3}\right)\left(\lambda_{2}-\lambda_{3}\right)} \\
& =\frac{1}{\prod_{1 \leq i<j \leq 3}\left(\lambda_{i}-\lambda_{j}\right)} \sum_{k=1}^{3} \lambda_{k}^{n} \cdot \prod_{i<j}^{i, j \neq k}\left(\lambda_{i}-\lambda_{j}\right)=\sum_{i=1}^{3} \frac{\lambda_{i}^{n}}{\prod_{i \neq j=1}^{3}\left(\lambda_{i}-\lambda_{j}\right)} .
\end{aligned}
$$

Due to the famous Cardano's method, the cubic equation $f(x)$ has roots

$$
\left\{\begin{array}{l}
\lambda_{1}=\frac{1}{3}\left(1+\tau+\frac{4}{\tau}\right)=\frac{1}{3}(1+X) \\
\lambda_{2}=\frac{1}{6}\left(2-\left(\tau+\frac{4}{\tau}\right)+i \sqrt{3}\left(\tau-\frac{4}{\tau}\right)\right)=\frac{1}{6}(2-X+i \sqrt{3} Y) \\
\lambda_{3}=\frac{1}{6}\left(2-\left(\tau+\frac{4}{\tau}\right)-i \sqrt{3}\left(\tau-\frac{4}{\tau}\right)\right)=\frac{1}{6}(2-X-i \sqrt{3} Y)
\end{array}\right.
$$

where $\tau=\sqrt[3]{19+3 \sqrt{33}}, \tau+\frac{4}{\tau}=X$ and $\tau-\frac{4}{\tau}=Y$. Then $\left(\lambda_{1}-\lambda_{2}\right)\left(\lambda_{1}-\lambda_{3}\right)=\frac{1}{3}\left(\left(\tau+\frac{4}{\tau}\right)^{2}-4\right)=\frac{1}{3}(X+2)(X-2)$ $\left(\lambda_{1}-\lambda_{2}\right)\left(\lambda_{2}-\lambda_{3}\right)=\frac{-1}{6}\left(\tau-\frac{4}{\tau}\right)\left(\left(\tau-\frac{4}{\tau}\right)+i \sqrt{3}\left(\tau+\frac{4}{\tau}\right)\right)=\frac{Y}{6}(Y+i \sqrt{3} X)$ $\left(\lambda_{1}-\lambda_{3}\right)\left(\lambda_{2}-\lambda_{3}\right)=\frac{-1}{6}\left(\tau-\frac{4}{\tau}\right)\left(\left(\tau-\frac{4}{\tau}\right)-i \sqrt{3}\left(\tau+\frac{4}{\tau}\right)\right)=-\frac{Y}{6}(Y-$ $i \sqrt{3} X)$

so

$\left(\lambda_{1}-\lambda_{2}\right)\left(\lambda_{1}-\lambda_{3}\right)\left(\lambda_{2}-\lambda_{3}\right)=i \frac{\sqrt{3}}{9}\left(\tau^{3}-\left(\frac{4}{\tau}\right)^{3}\right)=i \frac{\sqrt{3}}{9} 6 \sqrt{33}=i 2 \sqrt{11}$.

Thus

$$
A_{n}=\frac{-i}{2 \sqrt{11}}\left(\lambda_{1}^{n} \frac{i \sqrt{3}}{3} Y-\lambda_{2}^{n} \frac{3 X+i \sqrt{3} Y}{6}+\lambda_{3}^{n} \frac{3 X-i \sqrt{3} Y}{6}\right)
$$




$$
=\frac{\sqrt{3}}{12 \sqrt{11}}\left(2 \lambda_{1}^{n}-\lambda_{2}^{n}-\lambda_{3}^{n}\right)\left(\tau-\frac{4}{\tau}\right)+\frac{i}{4 \sqrt{11}}\left(\lambda_{2}^{n}-\lambda_{3}^{n}\right)\left(\tau+\frac{4}{\tau}\right) .
$$

Moreover since

$$
X^{3}=\left(\tau+\frac{4}{\tau}\right)^{3}=\tau^{3}+\left(\frac{4}{\tau}\right)^{3}+12\left(\tau+\frac{4}{\tau}\right)=38+12\left(\tau+\frac{4}{\tau}\right)=38+12 X
$$

and $Y^{3}=\left(\tau-\frac{4}{\tau}\right)^{3}=6 \sqrt{33}-12\left(\tau-\frac{4}{\tau}\right), X$ and $Y$ are real roots of $x^{3}-12 x-38=0$ and $x^{3}+12 x-6 \sqrt{33}=0$ respectively, so $X \sim 4.51$ and $Y \sim 2.10$. Thus $\lambda_{1}=\frac{1}{3}(1+X) \sim 1.84$ is a real root of $f(x)$ and the other $\lambda_{2}, \lambda_{3}$ are complex roots

$$
\frac{1}{6}(2-X \pm i \sqrt{3} Y) \sim \frac{1}{6}(-2.51 \pm i 2.10 \sqrt{3}) \sim-0.41 \pm 0.60 i .
$$

Hence as $n$ gets larger, $\lambda_{2}^{n}=\lambda_{3}^{n}$ goes to 0 , so

$$
A_{n} \sim \frac{\lambda_{1}^{n}}{\left(\lambda_{1}-\lambda_{2}\right)\left(\lambda_{1}-\lambda_{3}\right)}=\frac{\left(\frac{1}{3}\left(\tau+\frac{4}{\tau}+1\right)\right)^{n}}{\frac{1}{3}\left(\tau+\frac{4}{\tau}+2\right)\left(\tau+\frac{4}{\tau}-2\right)}=\frac{(X+1)^{n}}{3^{n}(X+2)(X-2)} .
$$

Theorem 7. Let $B_{n}=B_{n-1}+\cdots+B_{n-t}(n \geq t)$ with $B_{0}=\cdots=$ $B_{t-2}=0, B_{t-1}=1$. Then $B_{n}=\sum_{i=1}^{t} \frac{\lambda_{i}^{n}}{\prod_{i \neq j=1}^{t}\left(\lambda_{i}-\lambda_{j}\right)}$.

Proof. Let $t=4$. Then the characteristic polynomial $f(x)=x^{4}-$ $x^{3}-x^{2}-x-1$ of recurrence $\left\{B_{n}\right\}$ has 4 distinct roots $\lambda_{i}(1 \leq i \leq 4)$, two of them are real and the others are conjugate complex roots ([1]). So $B_{n}=\alpha_{1} \lambda_{1}^{n}+\alpha_{2} \lambda_{2}^{n}+\alpha_{3} \lambda_{3}^{n}+\alpha_{4} \lambda_{4}^{n}$ with $\alpha_{i} \in \mathbb{R}$ are determined by initial conditions $\left[\begin{array}{cccc}1 & 1 & 1 & 1 \\ \lambda_{1} & \lambda_{2} & \lambda_{3} & \lambda_{4} \\ \lambda_{1}^{2} & \lambda_{2}^{2} & \lambda_{3}^{2} & \lambda_{4}^{2} \\ \lambda_{1}^{3} & \lambda_{2}^{3} & \lambda_{3}^{3} & \lambda_{4}^{3}\end{array}\right]\left[\begin{array}{l}\alpha_{1} \\ \alpha_{2} \\ \alpha_{3} \\ \alpha_{4}\end{array}\right]=\left[\begin{array}{l}0 \\ 0 \\ 0 \\ 1\end{array}\right]$, thus due to the Gauss elimination we have $\alpha_{i}=\prod_{i \neq j=1}^{4} \frac{1}{\left(\lambda_{i}-\lambda_{j}\right)}$. Hence

$$
B_{n}=\sum_{i=1}^{4} \frac{\lambda_{i}^{n}}{\prod_{i \neq j=1}^{4}\left(\lambda_{i}-\lambda_{j}\right)}=\sum_{i=1}^{4} \frac{\lambda_{i}^{n} \prod_{1 \leq j<k \leq 4}^{i \neq j}\left(\lambda_{j}-\lambda_{k}\right)}{\prod_{1 \leq i<j \leq 4}\left(\lambda_{i}-\lambda_{j}\right)} .
$$

From the above formula of $B_{n}$ for $t=4$ as well as Theorem 6 for $t=3$, it is not hard to see the recurrence relation with $t$ predetermined terms forms $B_{n}=\sum_{i=1}^{t} \frac{\lambda_{i}^{n}}{\prod_{i \neq j=1}^{t}\left(\lambda_{i}-\lambda_{j}\right)}$. 


\section{Matrix formula distribution}

The formulas of the $n$th term $A_{n}$ discussed above is related to roots of polynomials. In this section we investigate more concrete method to compute $A_{n}$. Let us begin to distribute the Fibonacci sequence $F_{0}=$ $0, F_{1}=1, F_{2}=1, F_{3}=2, F_{4}=3, \cdots$ in rectangle form. May refer to [2] for Lucas sequence. We begin with 3 or 4 columns rectangle distribution.

\begin{tabular}{llll|llll}
\hline \multicolumn{3}{c||}{3 columns } & \multicolumn{5}{c}{4 columns } \\
\hline 1 & 1 & 2 & $\|$ & 1 & 1 & 2 & 3 \\
3 & 5 & 8 & $\|$ & 5 & 8 & 13 & 21 \\
13 & 21 & 34 & $\|$ & 34 & 55 & 89 & 144 \\
55 & 89 & 144 & $\|$ & 233 & 377 & 610 & 987 \\
233 & 377 & $\cdots$ & $\|$ & 1597 & 2584 & 4181 & $\cdots$
\end{tabular}

In the 3 columns distribution we find $233=(2 \cdot 2) 55+13$ and $377=$ $(2 \cdot 2) 89+21$. This means that

$$
F_{13}=2 F_{3} F_{10}+F_{7} \text { and } F_{14}=2 F_{3} F_{11}+F_{8} .
$$

Similarly from the 4 columns rectangle we have $4181=(2 \cdot 3+1) 610-89$, i.e,

$$
F_{19}=\left(2 F_{4}+F_{1}\right) F_{15}+(-1)^{3} F_{11} .
$$

Lemma 8. Any $n$th Fibonacci number $F_{n}$ satisfies $2 F_{3} F_{n}+F_{n-3}=$ $F_{n+3}$ and $\left(2 F_{4}+F_{1}\right) F_{n}+(-1)^{3} F_{n-4}=F_{n+4}$.

Proof. We first assume that $n \geq 4$. If $n=4$ then $2 F_{3} F_{4}+F_{1}=13=$ $F_{7}$. Assume $2 F_{3} F_{m}+F_{m-3}=F_{m+3}$ is true for all $m \leq n-1$. Then

$$
\begin{aligned}
2 F_{3} F_{n}+F_{n-3} & =2 F_{3}\left(F_{n-1}+F_{n-2}\right)+\left(F_{n-4}+F F_{n-5}\right) \\
& =\left(2 F_{3} F_{n-1}+F_{n-4}\right)+\left(2 F_{3} F_{n-2}+F_{n-5}\right) \\
& =F_{n+2}+F_{n+1} \\
& =F_{n+3} .
\end{aligned}
$$

Moreover by considering $F_{n}$ for negative $n$, such as $F_{-1}=1, F_{-2}=-1$, $F_{-3}=2, F_{-4}=-3, \cdots$, it is easy to see that the equality holds for all $n \in \mathbb{Z}$. Similarly the second identity also follows by induction that

$$
\begin{aligned}
\left(2 F_{4}\right. & \left.+F_{1}\right) F_{n}+(-1)^{3} F_{n-4} \\
& =\left(2 F_{4}+F_{1}\right)\left(F_{n-1}+F_{n-2}\right)+(-1)^{3}\left(F_{n-5}+F_{n-6}\right) \\
& =\left(\left(2 F_{4}+F_{1}\right) F_{n-1}+(-1)^{3} F_{n-5}\right)+\left(\left(2 F_{4}+F_{1}\right) F_{n-2}+(-1)^{3} F_{n-6}\right) \\
& =F_{n+3}+F_{n+2}=F_{n+4} .
\end{aligned}
$$

For instance, the 25th Fibonacci $F_{25}$ is, by the case of 3 columns rectangle,

$F_{25}=4 F_{22}+F_{19}=17 F_{19}+4 F_{16}=72 F_{16}+17 F_{13}=305 F_{13}+72 F_{10}$ 
$=1292 F_{10}+305 F_{7}=5473 F_{7}+1292 F_{4}=23184 F_{4}+5473 F_{1}=75025$. On the other hand, by considering 4 column rectangle, $F_{25}$ is $F_{25}=7 F_{21}-F_{17}=48 F_{17}-7 F_{13}=329 F_{13}-48 F_{9}=2255 F_{9}-329 F_{5}$

$=15456 F_{5}-2255 F_{1}=75025$.

We continue to consider 5 or 6 columns rectangle. The table with $k=5$

$\begin{array}{lllll}1 & 1 & 2 & 3 & 5 \\ 8 & 13 & 21 & 34 & 55 \\ 89 & 144 & 233 & 377 & 610 \\ 987 & 1597 & 2584 & 4181 & 6765 \\ 10946 & 17711 & 28657 & 46386 & \ldots\end{array}$

shows that $(2 \cdot 5+1)(987)+89=10946$, i.e. $\left(2 F_{5}+F_{2}\right) F_{16}+F_{11}=F_{21}$. And 6 columns distribution provides that $(2 \cdot 8+2) 28657-1597=514229$, i.e., $\left(2 F_{6}+F_{3}\right) F_{23}-F_{17}=F_{29}$. These observations yield the following theorem.

Theorem 9. Let $n \geq 1$ and $k \geq 3$ be given integers. Then

$$
F_{n+k}=\left(2 F_{k}+F_{k-3}\right) F_{n}+(-1)^{k-1} F_{n-k} .
$$

Moreover by dividing $n$ by $k$, let $n=Q k+r(Q, r \in \mathbb{Z})$ with $1 \leq r \leq k$. Then

$$
F_{n}=F_{Q k+r}=\left(2 F_{k}+F_{k-3}\right) F_{(Q-1) k+r}+(-1)^{k-1} F_{(Q-2) k+r} .
$$

Proof. Suppose $\left\{F_{n}\right\}$ is distributed in $k$ columns rectangle

$$
\begin{array}{llllll}
F_{1} & F_{2} & F_{3} & \cdots & F_{k-1} & F_{k} \\
F_{k+1} & F_{k+2} & F_{k+3} & \cdots & F_{k+(k-1)} & F_{2 k} \\
& \ddots & & \cdots & \ddots & \\
F_{t k+1} & F_{t k+2} & F_{t k+3} & \cdots & F_{t k+(k-1)} & F_{(t+1) k}
\end{array}
$$

It was proved that $F_{n+k}=\left(2 F_{k}+F_{k-3}\right) F_{n}+(-1)^{k-1} F_{n-k}$ for $k=3,4$ in Lemma 8. Assume this is true up to $k-1$. Then due to induction, we have

$F_{n+k}=F_{n+(k-1)}+F_{n+(k-2)}$

$=\left(2\left(F_{k-1}+F_{k-2}\right)+F_{k-4}+F_{k-5}\right) F_{n}+(-1)^{k-1}\left(-F_{n-(k-1)}+F_{n-(k-2)}\right)$

$=\left(2 F_{k}+F_{k-3}\right) F_{n}+(-1)^{k-1} F_{n-k}$.

Moreover if we write $n=Q k+r$ then

$$
\begin{aligned}
F_{Q k+r} & =F_{n}=F_{(n-k)+k}=\left(2 F_{k}+F_{k-3}\right) F_{n-k}+(-1)^{k-1} F_{n-2 k} \\
& =\left(2 F_{k}+F_{k-3}\right) F_{(Q-1) k+r}+(-1)^{k-1} F_{(Q-2) k+r} .
\end{aligned}
$$


It shows $F_{n}=F_{Q k+r}$ is a combination of $F_{(Q-1) k+r}$ and $F_{(Q-2) k+r}$. The following theorem will prove that $F_{n}$ is expressed by only $F_{k}, F_{k-3}$, $F_{r}$ and $F_{k+r}$.

Theorem 10. Under the same context above, if $n=Q k+r(1 \leq$ $r \leq k)$ then $F_{n}$ is a linear combination of $F_{k}, F_{k-3}, F_{r}$ and $F_{k+r}$.

Proof. Write $n=Q k+r$ with $Q, r \in \mathbb{Z}, 1 \leq r \leq k$. By Theorem 9,

$$
F_{Q k+r}=\mu F_{(Q-1) k+r}+(-1)^{k-1} F_{(Q-2) k+r}
$$

where $\mu=\left(2 F_{k}+F_{k-3}\right)$. For convenience we first assume $k$ is odd. Then

$F_{3 k+r}=\mu F_{2 k+r}+F_{k+r}=\mu\left(\mu F_{k+r}+F_{r}\right)+F_{k+r}=\left(\mu^{2}+1\right) F_{k+r}+\mu F_{r}$,

$F_{4 k+r}=\mu F_{3 k+r}+F_{2 k+r}=\left(\mu^{3}+2 \mu\right) F_{k+r}+\left(\mu^{2}+1\right) \mu F_{r}$.

Suppose it is true for each $t \leq Q-1$. Then the $t$ th stage can be written by

$$
F_{t k+r}=\alpha_{t} F_{k+r}+\beta_{t} F_{r}
$$

where $\alpha_{t}$ is $\mu$ times the first coefficient in the $(t-1)$ th stage added to the second coefficient in $t-1$ th stage, and $\beta_{t}$ is the first coefficient in $t-1$ th stage, that is,

$$
\alpha_{t}=\mu \alpha_{t-1}+\beta_{t-1} \text { and } \beta_{t}=\alpha_{t-1} .
$$

We will prove that $F_{Q k+r}=F_{(t+1) k+r}=\left(\mu \alpha_{t}+\beta_{t}\right) F_{k+r}+\alpha_{t} F_{r}$. Due to hypothesis we have $F_{(t-1) k+r}=\alpha_{t-1} F_{k+r}+\beta_{t-1} F_{r}$. Hence by Theorem 9 ,

$$
\begin{aligned}
F_{Q k+r} & =F_{(t+1) k+r}=\left(2 F_{k}+F_{k-3}\right) F_{t k+r}+F_{(t-1) k+r} \\
& =\mu\left(\alpha_{t} F_{k+r}+\beta_{t} F_{r}\right)+\alpha_{t-1} F_{k+r}+\beta_{t-1} F_{r} \\
& =\left(\mu \alpha_{t}+\alpha_{t-1}\right) F_{k+r}+\left(\mu \beta_{t}+\beta_{t-1}\right) F_{r} \\
& =\left(\mu \alpha_{t}+\beta_{t}\right) F_{k+r}+\left(\mu \alpha_{t-1}+\beta_{t-1}\right) F_{r}=\left(\mu \alpha_{t}+\beta_{t}\right) F_{k+r}+\alpha_{t} F_{r}
\end{aligned}
$$

as is desired. Since $\mu=\left(2 F_{k}+F_{k-3}\right), F_{n}$ is a combination of $F_{k}, F_{k-3}$, $F_{r}$ and $F_{k+r}$. The case for even $k$ also follows immediately.

Theorem 10 provides a good method to get $F_{n}$ by knowing a little information. Since $r$ and $k$ can be regarded as very smaller ones than $n, F_{n}$ can be obtained by only relatively small four Fibonacci numbers $F_{k}, F_{k-3}, F_{r}$ and $F_{k+r}$.

Example 1. For the $F_{50}$, we may take any $k$, say for instance $k=7$. Then

$F_{50}=F_{7 \cdot 7+1}=\mu F_{6 \cdot 7+1}+F_{5 \cdot 7+1}$

$=\left(\mu^{2}+1\right) F_{5 \cdot 7+1}+\mu F_{4 \cdot 7+1}$

$=\left(\mu\left(\mu^{2}+1\right)+\mu\right) F_{4 \cdot 7+1}+\left(\mu^{2}+1\right) F_{3 \cdot 7+1}$

$=\left(\mu\left(\mu^{3}+2 \mu\right)+\mu^{2}+1\right) F_{3 \cdot 7+1}+\left(\mu^{3}+2 \mu\right) F_{2 \cdot 7+1}$

$=\left(\mu\left(\mu^{4}+3 \mu^{2}+1\right)+\mu^{3}+2 \mu\right) F_{2 \cdot 7+1}+\left(\mu^{4}+3 \mu^{2}+1\right) F_{7+1}$ 
$=\left(\mu\left(\mu^{5}+4 \mu^{3}+3 \mu\right)+\mu^{4}+3 \mu^{2}+1\right) F_{7+1}+\left(\mu^{5}+4 \mu^{3}+3 \mu\right) F_{1}=$ $12,586,269,025$

because $F_{7}=13, F_{4}=3, F_{1}=1, F_{8}=21$ and $\mu=2 F_{7}+F_{4}=29$.

Regarding the above $k$ columns rectangle as a matrix with $k$ columns, we may consider, for instance, $F_{k+3}$ as the entry $\mathcal{E}_{(2,3)}$ at the 2nd row and 3rd column, and in general we may consider $F_{(Q-1) k+r}=\mathcal{E}_{(Q, r)}$ as the entry at $Q$ th row and $r$ th column. Then the above theorem implies that, when $n=Q k+r$

$$
F_{n}=\mathcal{E}_{(Q+1, r)}=\mu \mathcal{E}_{(Q, r)}+(-1)^{k-1} \mathcal{E}_{(Q-1, r)}
$$

with $\mu=2 \mathcal{E}_{(1, k)}+\mathcal{E}_{(1, k-3)}$. It shows that $F_{n}$ is a linear combination of two preceding entries $\mathcal{E}_{(Q, r)}$ and $\mathcal{E}_{(Q-1, r)}$ located in the same column that $F_{n}$ belongs.

Corollary 11. $F_{n}$ is a linear combination of three entries $\mathcal{E}_{(1, k)}$, $\mathcal{E}_{(1, k-3)}$ and $\mathcal{E}_{(1, r)}$ in the first row, and one entry $\mathcal{E}_{(1, k+r)}$ in the second row.

We shall extend this investigation to the 3 -step tribonacci sequence $T_{n}$ such that $T_{1}=0, T_{2}=1, T_{3}=1, T_{4}=2, T_{5}=4, T_{6}=7, \cdots$. We begin with 4 or 5 columns.

\begin{tabular}{llll||llllll}
\hline \multicolumn{3}{c}{4 columns } & \multicolumn{7}{c}{5 columns } \\
\hline 1 & 1 & 2 & 4 & $\|$ & 1 & 1 & 2 & 4 & 7 \\
7 & 13 & 24 & 44 & $\|$ & 13 & 24 & 44 & 81 & 149 \\
81 & 149 & 274 & 504 & $\|$ & 274 & 504 & 927 & 1705 & 3136 \\
927 & 1705 & 3136 & 5768 & $\|$ & 5768 & 10609 & 19513 & 35890 & 66012 \\
10609 & 19513 & 35890 & $\ldots$ & $\|$ & 121415 & 223317 & 410744 & $\ldots$
\end{tabular}

It is clear that, in 4 columns rectangle

$T_{16}=5768=11 \cdot 504+5 \cdot 44+4=\left(3 T_{4}-1\right) T_{12}+\left(T_{4}+1\right) T_{8}+T_{4}$,

$T_{19}=35890=11 \cdot 3136+5 \cdot 274+24=\left(3 T_{4}-1\right) T_{15}+\left(T_{4}+1\right) T_{11}+T_{7}$.

Also in 5 columns, $T_{17}=10690=21 \cdot 504+24+1=\left(3 T_{5}\right) T_{12}+T_{7}+T_{2}$, and

$T_{23}=410744=21 \cdot 19513+927+44=\left(3 T_{5}\right) T_{18}+T_{13}+T_{6}$.

Moreover considering the 6 columns rectangle we can find

$T_{22}=223317=39 \cdot 5768-(13-2) 149+4=\left(3 T_{6}\right) T_{16}-\left(T_{6}-2\right) T_{10}+T_{4}$.

These considerations would yield the following theorem.

Theorem 12. Let $n=k Q+r$ with $1 \leq r \leq k$. Then for $4 \leq k \leq 9$

$$
T_{k Q+r}=\mu_{1} T_{k(Q-1)+r}+\mu_{2} T_{k(Q-2)+r}+\mu_{3} T_{k(Q-3)+r}
$$


where the coefficients $\left(\mu_{1}, \mu_{2}, \mu_{3}\right)$ are determined as follows.

\begin{tabular}{|l|l||c|l||c|l|}
\hline$k$ & $\left(\mu_{1}, \mu_{2}, \mu_{3}\right)$ & $k$ & $\left(\mu_{1}, \mu_{2}, \mu_{3}\right)$ & $k$ & $\left(\mu_{1}, \mu_{2}, \mu_{3}\right)$ \\
\hline 4 & $\left(3 T_{4}-1, T_{4}+1,1\right)$ & 5 & $\left(3 T_{5}, 1,1\right)$ & 6 & $\left(3 T_{6},-T_{6}+2,1\right)$ \\
\hline 7 & $\left(3 T_{7}-1,15,1\right)$ & 8 & $\left(3 T_{8}-1,-1,1\right)$ & 9 & $\left(3 T_{9}-2,-23,1\right)$ \\
\hline
\end{tabular}

Proof. The above observations prove this for $4 \leq k \leq 6$. When $k=7$,

$\begin{array}{lllllll}1 & 1 & 2 & 4 & 7 & 13 & 24 \\ 44 & 81 & 149 & 274 & 504 & 927 & 1705 \\ 3136 & 5768 & 10609 & 19513 & 35890 & 66012 & 121415 \\ 223317 & 410744 & 755476 & 1389537 & 2555757 & 4700770 & \ldots\end{array}$

it is easy to see that

$T_{22}=223317=71 \cdot 3136+15 \cdot 44+1=\left(3 T_{7}-1\right) T_{15}+15 T_{8}+T_{1}$.

Similarly for $k=8,9$ and 10 , we see that

$T_{25}=1389537=131 \cdot 10609-3 \cdot 81+1=\left(3 T_{8}-1\right) T_{17}-3 T_{9}+T_{1}$,

$T_{33}=241 \cdot 755476-23 \cdot 3136+13=\left(3 T_{9}-2\right) T_{24}-(23) T_{15}+T_{6}$,

$T_{31}=443 \cdot 121415+41 \cdot 274+1=\left(3 T_{10}-4\right) T_{21}+(41) T_{11}+T_{1}$

Based on these observation, the proof follows by induction. For instance the observation $\left(3 T_{9}-2\right) T_{24}-(23) T_{15}+T_{6} T_{33}$ shows

$$
\left(3 T_{k}-2\right) T_{m}-23 T_{m-k}+T_{m-2 k}=T_{m+k} \quad \text { with } k=9, m=24 .
$$

We assume the identity is true for each $m \leq n$. Then

$$
\left(3 T_{k}-2\right) T_{n+1}-23 T_{(n+1)-k}+T_{(n+1)-2 k}
$$

$=\left(3 T_{k}-2\right)\left(T_{n}+T_{n-1}+T_{n-2}\right)-23\left(T_{n-k}+T_{n-k-1}+T_{n-k-2}\right)$

$$
+\left(T_{n-2 k}+T_{n-2 k-1}+T_{n-2 k-2}\right)
$$

$=\left(\left(3 T_{k}-2\right) T_{n}-23 T_{n-k}+T_{n-2 k}\right)+\left(\left(3 T_{k}-2\right) T_{n-1}-23 T_{(n-1)-k}+\right.$ $\left.T_{(n-1)-2 k}\right)$

$$
+\left(\left(3 T_{k}-2\right) T_{n-2}-23 T_{(n-2)-k}+T_{(n-2)-2 k}\right)
$$

$=T_{n+k}+T_{(n-1)+k}+T_{(n-2)+k}=T_{n+k+1}=T_{(n+1)+k}$.

Therefore for $k=9$ and $n=k Q+r$ with $1 \leq r \leq k$, it follows that

$$
\begin{aligned}
T_{k Q+r} & =T_{n}=T_{(n-k)+k} \\
& =\left(3 T_{k}-2\right) T_{n-k}-23 T_{(n-k)-k}+T_{(n-k)-2 k} \\
& =\left(3 T_{k}-2\right) T_{k(Q-1)+r}-23 T_{k(Q-2)+r}+T_{k(Q-3)+r}
\end{aligned}
$$

as is desired. For the other $k$, the proof follows similarly.

The formula for the coefficients that represent $F_{n}$ as linear combination in Theorem 9 are the same no matter we choose any $k$, but the form for the coefficient that represent $T_{n}$ in Theorem 12 vary depending on $k$. 

write

Regarding $T_{Q k+r}$ as an entry $\mathcal{E}_{(Q+1, r)}$ in $k$-columns rectangle, we can

$$
T_{n}=\mathcal{E}_{(Q+1, r)}=\mu_{1} \mathcal{E}_{(Q, r)}+\mu_{2} \mathcal{E}_{(Q-1, r)}+\mu_{3} \mathcal{E}_{(Q-2, r)}
$$

where the coefficient $\left(\mu_{1}, \mu_{2}, \mu_{3}\right)$ forms as follows:

\begin{tabular}{|l|l||l|l||c|l|}
\hline$k$ & $\left(\mu_{1}, \mu_{2}, \mu_{3}\right)$ & $k$ & $\left(\mu_{1}, \mu_{2}, \mu_{3}\right)$ & $k$ & $\left(\mu_{1}, \mu_{2}, \mu_{3}\right)$ \\
\hline 4 & $\left(3 \mathcal{E}_{(1,4)}-1,3 \mathcal{E}_{(1,4)}+1,1\right)$ & 5 & $\left(3 \mathcal{E}_{(1,5)}, 1,1\right)$ & 6 & $\left(3 \mathcal{E}_{(1,6)},-\mathcal{E}_{(1,6)}+2,1\right)$ \\
\hline 7 & $\left(3 \mathcal{E}_{(1,7)}-1,15,1\right)$ & 8 & $\left(3 \mathcal{E}_{(1,8)}-1,-1,1\right)$ & 9 & $\left(3 \mathcal{E}_{(1,9)}-2,-23,1\right)$ \\
\hline
\end{tabular}

As an analogue of Theorem 10 or Corollary 11, we are able to write $T_{n}=T_{Q k+r}$ as a combination of $\mathcal{E}_{(3, r)}, \mathcal{E}_{(2, r)}$ and $\mathcal{E}_{(1, r)}$. In fact

$T_{Q k+r}=\mathcal{E}_{(Q+1, r)}$

$=\mu_{1} \mathcal{E}_{(Q, r)}+\mu_{2} \mathcal{E}_{(Q-1, r)}+\mu_{3} \mathcal{E}_{(Q-2, r)}$

$=\mu_{1}\left(\mu_{1} \mathcal{E}_{(Q-1, r)}+\mu_{2} \mathcal{E}_{(Q-2, r)}+\mu_{3} \mathcal{E}_{(Q-3, r)}\right)+\mu_{2} \mathcal{E}_{(Q-1, r)}+\mu_{3} \mathcal{E}_{(Q-2, r)}$

$=\left(\mu_{1}^{2}+\mu_{2}\right) \mathcal{E}_{(Q-1, r)}+\left(\mu_{1} \mu_{2}+\mu_{3}\right) \mathcal{E}_{(Q-2, r)}+\mu_{1} \mathcal{E}_{(Q-3, r)}$

$=\left(\mu_{1}^{2}+\mu_{2}\right)\left(\mu_{1} \mathcal{E}_{(Q-2, r)}+\mu_{2} \mathcal{E}_{(Q-3, r)}+\mu_{3} \mathcal{E}_{(Q-4, r)}\right)+\left(\mu_{1} \mu_{2}+\mu_{3}\right) \mathcal{E}_{(Q-2, r)}+$ $\mu_{1} \mathcal{E}_{(Q-3, r)}$

$=\left(\mu_{1}\left(\mu_{1}^{2}+\mu_{2}\right)+\left(\mu_{1} \mu_{2}+\mu_{3}\right)\right) \mathcal{E}_{(Q-2, r)}+\left(\mu_{2}\left(\mu_{1}^{2}+\mu_{2}\right)+\mu_{3}\right) \mathcal{E}_{(Q-3, r)}+$ $\left(\mu_{1}^{2}+\mu_{2}\right) \mu_{3} \mathcal{E}_{(Q-4, r)}$.

Hence after some stages, if we write

$$
T_{Q k+r}=\theta_{1} \mathcal{E}_{(Q-s, r)}+\theta_{2} \mathcal{E}_{(Q-(s+1), r)}+\theta_{3} \mathcal{E}_{(Q-(s+2), r)}
$$

then $T_{Q k+r}$ in the next step can be written as

$T_{Q k+r}=\left(\mu_{1} \theta_{1}+\theta_{2}\right) \mathcal{E}_{(Q-(s+1), r)}+\left(\mu_{2} \theta_{1}+\theta_{3}\right) \mathcal{E}_{(Q-(s+2), r)}+\mu_{3} \theta_{1} \mathcal{E}_{(Q-(s+3), r)}$.

The $k$ could be any integer. But if $k$ is large then the $r$ in $Q k+r$ could be also large, so that $T_{k}$ and $T_{r}$ themselves are too big to be used for $T_{n}$.

Example 2. For $T_{50}$, if we take $k=7$ then with the coefficient $\left(\mu_{1}, \mu_{2}, \mu_{3}\right)=\left(3 \mathcal{E}_{(1,7)}-1,15,1\right)=(71,15,1)$, it follows inductively that $T_{50}=\mathcal{E}_{(8,1)}=71 \mathcal{E}_{(7,1)}+15 \mathcal{E}_{(6,1)}+\mathcal{E}_{(5,1)}$

$=(71 \cdot 71+15) \mathcal{E}_{(6,1)}+(15 \cdot 71+1) \mathcal{E}_{(5,1)}+71 \mathcal{E}_{(4,1)}$

$=360042 \mathcal{E}_{(5,1)}+75911 \mathcal{E}_{(4,1)}+5056 \mathcal{E}_{(3,1)}$

$=25638893 \mathcal{E}_{(4,1)}+5405686 \mathcal{E}_{(3,1)}+360042 \mathcal{E}_{(2,1)}$

$=1825767089 \mathcal{E}_{(3,1)}+384943437 \mathcal{E}_{(2,1)}+25638893 \mathcal{E}_{(1,1)}$

$=5,742,568,741,225$

because $\mathcal{E}_{(3,1)}=3136, \mathcal{E}_{(2,1)}=44$ and $\mathcal{E}_{(1,1)}=1$ in 7 columns distribution. 


\section{References}

[1] M. Abramowitz, I. Stegun (Eds.), Solutions of Quartic Equations, Handbook of Mathematical Functions with Formulas, Graphs, and Mathematical Tables, New York: Dover, 17-18, 1972.

[2] H. W. Austin, Columns of Fibonacci or Lucas Numbers, Mathematical Spectrum, 37 (2005), 67-72.

[3] H. Cohen, Resultants and discriminants. A Course in Computational Algebraic Number Theory, New York, Springer Verlag, 119-123, 1993.

[4] K.Rosen, Discrete Mathematics and its applications. Boston, McGraw Hill, 414415, 2003.

\section{Eunmi Choi}

Department of Mathematics, Hannam University, Daejon 306-791, Korea.

E-mail: emc@hnu.kr 